\title{
Length of stay in surgical patients: nutritional predictive parameters revisited
}

\author{
Ana Isabel Almeida, Marta Correia, Maria Camilo and Paula Ravasco* \\ Unidade de Nutrição e Metabolismo, Instituto de Medicina Molecular, Laboratório de Nutrição, Faculdade de Medicina \\ da Universidade de Lisboa, \\ Avenida Professor Egas Moniz, 1649-028 Lisboa, Portugal
}

(Submitted 22 November 2011 - Final revision received 24 February 2012 - Accepted 24 February 2012 - First published online 1 May 2012)

\begin{abstract}
Nutritional evaluation may predict clinical outcomes, such as hospital length of stay (LOS). We aimed to assess the value of nutritional risk and status methods, and to test standard anthropometry percentiles $v$. the 50th percentile threshold in predicting LOS, and to determine nutritional status changes during hospitalisation and their relation with LOS. In this longitudinal prospective study, 298 surgical patients were evaluated at admission and discharge. At admission, nutritional risk was assessed by Nutritional Risk Screening-2002 (NRS-2002), Malnutrition Universal Screening Tool (MUST) and nutritional status by Subjective Global Assessment (SGA), involuntary \% weight loss in the previous 6 months and anthropometric parameters; \% weight loss and anthropometry were reassessed at discharge. At admission, risk/undernutrition results by NRS-2002 ( $P<0 \cdot 001)$, MUST $(P<0 \cdot 001)$, \% weight loss $(P<0 \cdot 001)$ and SGA $(P<0 \cdot 001)$ were predictive of longer LOS. A mid-arm circumference (MAC) or a mid-arm muscle circumference (MAMA) under the 15th and the 50th percentile, which was considered indicative of undernutrition, did predict longer LOS $(P<0 \cdot 001)$; conversely, there was no association between depleted triceps skinfold (TSF) and longer LOS. In-hospital, there was a high prevalence of weight, muscle and fat losses, associated with longer LOS. At discharge, patients with a simultaneous negative variation in TSF + MAC + MAMA ( $n$ 158, 53\%) had longer LOS than patients with a TSF + MAC + MAMA positive variation $(11(8-15) v .8(7-12) \mathrm{d}, P<0 \cdot 001)$. We concluded that at risk or undernutrition evaluated by all methods, except TSF and BMI, predicted a longer LOS. Moreover, MAC and MAMA measurements and their classification according to the 50th percentile threshold seem reliable undernutrition indicators.
\end{abstract}

\section{Key words: Surgery: Length of stay: Nutritional risk: Nutritional status: Nutritional deterioration: Predictive values}

In hospitalised surgical patients, undernutrition remains widely undiagnosed/underestimated, with a prevalence of $40-64 \%$ depending on the criteria used ${ }^{(1-4)}$. Undernutrition has been associated with impaired wound healing and higher infection rates, increased postoperative morbidity and mortality, longer hospital length of stay (LOS) and increased overall costs ${ }^{(5-7)}$.

Nutritional screening on admission to detect patients who may require early nutritional intervention is recognised $^{(8)}$; recent evidence advocates that screening and assessment methods may also predict clinical outcomes, such as hospital $\operatorname{LOS}^{(9-12)}$. Moreover, since surgery induces catabolism not always compensated by appropriate nutritional support, surgical patients are more prone to nutritional deterioration throughout their hospital sojourn ${ }^{(13)}$; however, few studies ${ }^{(1,14-16)}$ have evaluated such changes. Weight loss and anthropometry may offer a more correct approach to quantify in-hospital nutritional depletion, although current anthropometric thresholds may possibly be deficient in detecting undernutrition in clinical practice ${ }^{(15,16)}$
The aims of this longitudinal study conducted in hospitalised surgical patients were: (1) to assess the value of nutritional risk (Nutritional Risk Screening-2002 (NRS-2002), Malnutrition Universal Screening Tool (MUST)) and nutritional status methods (recent \% weight loss, Subjective Global Assessment (SGA), anthropometry) in predicting hospital LOS; (2) to test the ability of current percentiles thresholds in detecting undernutrition and predicting LOS and (3) to determine possible changes in nutritional status during hospitalisation and their association with LOS.

\section{Material and methods}

\section{Study population}

This prospective longitudinal study was carried out in a University Hospital large general Surgical Department in Lisbon, Portugal. The study was approved by the Ethics Committee and was conducted in accordance with the Helsinki Declaration

Abbreviations: GI, gastrointestinal; LOS, length of stay; MAC, mid-arm circumference; MAMA, mid-arm muscle area; MUST, Malnutrition Universal Screening Tool; NRS-2002, Nutritional Risk Screening-2002; SGA, Subjective Global Assessment; TSF, triceps skinfold. 
of 1975 as revised in 1983 and 2002; included patients gave their written informed consent to participate. During 8 months, all consecutive adult patients ( $\geq 18$ years) with a predicted length of stay $\geq 4 \mathrm{~d}$ were considered eligible. At admission, 300 patients met the inclusion criteria. Exclusion criteria comprised organ transplantation, coma, bedridden, intensive care patients, or previous surgery, chemo/radiotherapy during the year before hospital admission; patients submitted to surgery before nutritional assessment were also excluded ( $n$ 25). Since two patients died before the second evaluation, 298 patients were longitudinally assessed at admission and discharge. Patients' characteristics are summarised in Table 1.

\section{Study parameters}

All nutritional data were collected by a single research dietitian (M. Correia). Nutritional risk and status were assessed before any surgical procedure and within $3 \mathrm{~d}$ after hospital admission; weight loss and anthropometric data were again evaluated $24 \mathrm{~h}$ before discharge.

\section{Nutritional risk}

NRS-2002 screens patients by grading nutritional status and disease severity (low, moderate or severe), with an adjustment for age $\geq 70$ years; a patient is at nutritional risk when the final score is $\geq 3^{(17)}$. MUST integrates BMI, unintentional recent weight loss and scores the acute disease effect on intake. Nutritional risk is then categorised: $0=$ low risk, $1=$ medium risk and $\geq 2=$ high risk $^{(18)}$. In order to enable comparisons between methods, MUST categories were grouped in: low $v$. moderate + high risk.

\section{Nutritional status}

SGA is based on the patient's clinical history, physical examination, estimated weight loss and changes in diet intake allowing nutritional status categorisation: (a) well nourished, (b) moderately undernourished or suspected of being undernourished, and (c) severely undernourished ${ }^{(19)}$.

Percentage weight loss was calculated and classified at admission as clinically significant, indicative of undernutrition or of nutritional

Table 1. Patients' characteristics

(Number of patients and percentages)

\begin{tabular}{|c|c|c|c|c|c|c|}
\hline & \multicolumn{2}{|c|}{ Total } & \multicolumn{2}{|c|}{$\begin{array}{l}\text { Cancer } \\
(n 138)\end{array}$} & \multicolumn{2}{|c|}{$\begin{array}{c}\text { Non-cancer } \\
(n 160)\end{array}$} \\
\hline & $n$ & $\%$ & $n$ & $\%$ & $n$ & $\%$ \\
\hline Men & 132 & 44 & 61 & 20 & 71 & 24 \\
\hline Women & 166 & 56 & 77 & 26 & 89 & 30 \\
\hline \multicolumn{7}{|l|}{ Age (years) } \\
\hline Mean & \multicolumn{2}{|c|}{60} & \multicolumn{2}{|c|}{63} & \multicolumn{2}{|c|}{57} \\
\hline SD & \multicolumn{2}{|c|}{17} & \multicolumn{2}{|c|}{15} & \multicolumn{2}{|c|}{18} \\
\hline$\geq 65$ years & 132 & 44 & 69 & 23 & 63 & 21 \\
\hline Elective surgery & 162 & 54 & 84 & 28 & 78 & 26 \\
\hline Non-elective surgery & 136 & 46 & 54 & 18 & 82 & 28 \\
\hline Gl surgery & 227 & 76 & 102 & 34 & 125 & 42 \\
\hline Non-Gl surgery & 71 & 24 & 36 & 12 & 35 & 12 \\
\hline
\end{tabular}

GI, gastrointestinal. risk, if $\geq 5 \%$ in the previous 6 months $^{(8)}$; $\%$ weight loss was determined at discharge and valued by itself or cumulatively.

Anthropometric data included height, weight, triceps skinfold thickness (TSF), mid-arm circumference (MAC) and midarm muscle area (MAMA). Height was measured in the upright position using a stadiometer, and weight was determined with a Seca floor scale and registered to the nearest $0.5 \mathrm{~kg}$; both allowed the calculation of BMI $(\mathrm{BMI}=$ weight $(\mathrm{kg}) /$ height $\left(\mathrm{m}^{2}\right)$ ). BMI was classified using the WHO criteria: underweight if $<18.5 \mathrm{~kg} / \mathrm{m}^{2}$, regular $18.5-24.9 \mathrm{~kg} / \mathrm{m}^{2}$, overweight $25-29.9 \mathrm{~kg} / \mathrm{m}^{2}$ or obese $\geq 30 \mathrm{~kg} / \mathrm{m}^{2(20)}$. TSF in $\mathrm{mm}$ was measured with a skinfold calliper ( John Bull ${ }^{\circledR}$; Briish Indicators Ltd) at the back of the non-dominant arm, at the midpoint between the tip of the acromial process of the scapula and the olecraneon process of the ulna; the mean of three readings was used. MAC in cm was measured at the same site and position as described for TSF perpendicular to the long axis of the arm, using a non-stretchable flexible tape; care was taken not to pinch or gap the tape and three measurements to the nearest $0 \cdot 1 \mathrm{~cm}$ were taken and their mean was used. MAMA in $\mathrm{cm}^{2}$ was calculated using TSF and MAC values, according to the formula:

$$
\operatorname{MAMA}=\left(\operatorname{MAC}(\mathrm{cm})-0.314 \times \operatorname{TSF}(\mathrm{mm})^{2}\right) /(4 \times 3 \cdot 14) .
$$

The calculated muscle area was adjusted for bone area by subtracting $6.5 \mathrm{~cm}^{2}$ in women and $10 \mathrm{~cm}^{2}$ in men. Reference tables, standardised for age and sex, and validated for normal subjects were used for the classification of individual values $^{(21)}$; a TSF, MAC or MAMA $<15$ th percentile is indicative of undernutrition, whereas a TSF $>85$ th percentile is indicative of excess body fat ${ }^{(21)}$.

\section{Statistical analysis}

Data were analysed using SPSS 16.0 statistical software (SPSS, Inc.). Categorical data were expressed as number and percentage of patients; continuous data with a normal distribution were expressed as means and standard deviations, while continuous data not normally distributed were expressed as medians with their interquartile range. Comparisons were made using non-parametric tests as appropriate: Wilcoxon $W$ for comparing median values between two independent groups; median test for comparing median values between three or more groups and the $\chi^{2}$ test for comparing categorical data. Spearman's correlation coefficient was calculated to verify linear association between two continuous variables. Agreement analysis between methods and LOS was performed using the concordance $\kappa$ coefficient, the statistics of which were further classified according to Fleiss ${ }^{(22)}$. For $\kappa$ coefficient calculation, two categories of LOS were established by using the median value of its distribution ( $10 \mathrm{~d}$ ) as the cut-off value.

Sensitivity, specificity and predictive values were calculated to appraise the value of the nutritional risk and status tools tested in predicting LOS. A prolonged LOS was defined as a stay in hospital above the median value of its distribution. Sensitivity was expressed as the proportion of patients with a longer LOS, which were classified as 'at nutritional risk' or 'undernourished' or 'underweight' by nutritional methods. On the other hand, specificity refers to the proportion of patients 
with a LOS below the 50th percentile and classified as 'without nutritional risk' or 'well nourished' or 'well nourished/ overweight/obese' by nutritional methods. The positive predictive value is defined as the proportion of patients classified as 'at nutritional risk' or 'undernourished' or 'underweight' by nutritional methods, which have a longer LOS. Conversely, the negative predictive value is the proportion of patients identified as 'without nutritional risk' or 'well nourished/overweight/obese' by nutritional methods, who have a LOS below the 50 th percentile.

Statistical significance was determined for $P<0.05$.

\section{Results}

The median hospital LOS was $10(7-14) \mathrm{d}$; significantly longer in male $(11(8-14) v .8(7-13) \mathrm{d}, P<0.001)$ and in patients $\geq 65$ years $(11(8-15) v \cdot 9(7-12) \mathrm{d}, P<0 \cdot 01)$. According to diagnoses, cancer patients had a longer LOS than non-cancer patients (11 (8-14) v. $9(7-12.5) \mathrm{d}, P<0 \cdot 01)$; among those, gastrointestinal (GI) cancer patients ( $n 99,33 \%$ ) stayed in hospital for a longer period (12 $(9-16) d)$, when compared with breast or gynaecological cancer patients ( $n 28,9 \%$; 7 (6-8) d, $P<0 \cdot 001$ ). Regarding indication for surgery, the median LOS of patients admitted for non-elective surgery was significantly longer than that of patients admitted for elective surgery (11 (8-16) v. $8.5(7-12) \mathrm{d}, P<0.001)$. LOS was also longer in GI surgery $v$. non-GI surgery $(11(8-15) v .7(6-8.5) \mathrm{d}, P<0.001)$.

\section{Admission}

Length of stay in relation to nutritional risk and status methods. The ability of the various methods to predict hospital LOS was the first step in the analyses (Table 2).

Nutritional Risk Screening-2002 and Malnutrition Universal Screening Tool. Patients identified as at nutritional risk by either NRS-2002 or MUST categories had a longer median LOS (risk $v$. low risk for NRS-2002 and moderate + high risk $v$. low risk for MUST, $P<0 \cdot 001$.

Percentage weight loss, Subjective Global Assessment and $B M I$. Patients who reported $\geq 5 \%$ weight loss at admission were hospitalised for a longer period $(P<0 \cdot 001)$. According to SGA, LOS was significantly longer in severe and moderate undernutrition $v$. well-nourished patients $(P<0 \cdot 001)$. Using BMI, hospital LOS was longer in underweight than in regularweight patients $(P<0.05)$, in the latter median LOS was not significantly different from overweight/obese.

Anthropometry. Using the previous anthropometric percentile criteria mentioned in the Methods section defining undernutrition $^{(21)}$, there was a lower prevalence of undernourished patients when compared with SGA; $69-75 \%$ of patients identified as undernourished by SGA were classified as well nourished by anthropometric criteria, which may signify a misclassification by anthropometry. Moreover, $\kappa$ coefficient agreement between the standard thresholds of TSF/MAC/ MAMA $v$. SGA was low $(\kappa=0 \cdot 15-0 \cdot 18, P<0 \cdot 01)$. Based on the poor performance of the standard percentile thresholds (15th and 85th percentiles) in predicting hospital LOS, we thus tested a unique and simpler percentile value (50th percentile) in order to evaluate its clinical feasibility (Table 3).

Using the standard percentiles, a TSF $\leq 15$ th did not predict a longer LOS $v$. a TSF within 15th-85th percentiles; however, patients with a TSF $\geq 85$ th percentile stayed longer in-hospital $(P<0.05)$ than patients with a TSF between the 15 th and 85 th percentiles. Conversely, a MAC or MAMA $\leq 15$ th percentile predicted a longer LOS $(P<0 \cdot 001)$, whereas patients with MAMA $\geq 85$ th percentile had a shorter hospital stay $(P<0 \cdot 05)$. Furthermore, the simultaneous presence of TSF + MAC + MAMA $\leq 15$ th percentile did not predict a longer LOS $v$. patients with TSF + MAC + MAMA $>15$ th percentile $(12(9-22 \cdot 5) v .10(7-13) \mathrm{d}, \mathrm{NS})$.

Table 2. Median length of stay (LOS) according to nutritional risk and status at admission (Number of patients and percentages; medians and interquartile ranges (IQR))

\begin{tabular}{|c|c|c|c|c|c|}
\hline \multirow[b]{2}{*}{ Nutritional risk and status } & \multirow[b]{2}{*}{$n$} & \multirow[b]{2}{*}{$\%$} & \multicolumn{2}{|c|}{$\operatorname{LOS}(d)$} & \multirow[b]{2}{*}{$P$} \\
\hline & & & Median & IQR & \\
\hline NRS-2002 & & & & & $<0.001^{*}$ \\
\hline Low risk & 101 & 34 & 7 & $6-10$ & \\
\hline Risk & 197 & 66 & 11 & $8-15$ & \\
\hline MUST & & & & & $<0.001^{*}$ \\
\hline Low risk & 103 & 35 & 8 & $6 \cdot 5-10$ & \\
\hline Medium + high risk & 195 & 65 & 12 & $8-16$ & \\
\hline Recent weight loss (kg) & & & & & $<0.001^{*}$ \\
\hline$<5 \%$ & 115 & 39 & 8 & $6-10$ & \\
\hline$\geq 5 \%$ (undernutrition) & 183 & 61 & 11 & $8-16$ & \\
\hline SGA & & & & & $<0.001 \dagger$ \\
\hline Well nourished & 107 & 36 & 8 & $6-10$ & \\
\hline Moderate undernutrition & 106 & 36 & 10 & $8-14$ & \\
\hline Severe undernutrition & 85 & 28 & 12 & $10-14$ & \\
\hline BMI $\left(\mathrm{kg} / \mathrm{m}^{2}\right)$ & & & & & NS† \\
\hline Underweight & 17 & 6 & 12 & $9-17$ & \\
\hline Normal weight & 191 & 64 & 9 & $7-14$ & \\
\hline Overweight/obesity & 90 & 30 & 9.5 & $7-13$ & \\
\hline
\end{tabular}

NRS-2002, Nutritional Risk Screening-2002; MUST, Malnutrition Universal Screening Tool; SGA, Subjective Global Assessment. Statistical analysis was performed with non-parametric * Wilcoxon $W$ test or non-parametric; $†$ Median $T$ test as appropriate, for median values comparison. $P$ values express statistical differences between LOS in each nutritional risk and status category. 
Table 3. Median length of stay (LOS) using standard percentiles ( $\leq 15$ th, 15 th -85 th, $\geq 85$ th) $v$. a proposed threshold $(<50$ th, $\geq 50$ th percentile) for anthropometry at admission and median variation of triceps skinfold (TSF), mid-arm circumference (MAC) and mid-arm muscle area (MAMA) during hospital stay

(Number of patients and percentages; medians and interquartile ranges (IQR))

\begin{tabular}{|c|c|c|c|c|c|c|c|c|c|}
\hline \multirow[b]{3}{*}{ Nutritional status } & \multicolumn{4}{|c|}{ Admission } & \multirow[b]{3}{*}{$P \neq$} & \multirow{3}{*}{\multicolumn{2}{|c|}{$\Delta$ Discharge-admission }} & & \multirow[b]{3}{*}{$P \S$} \\
\hline & \multirow[b]{2}{*}{$n$} & \multirow[b]{2}{*}{$\%$} & \multicolumn{2}{|c|}{$\operatorname{LOS}(d)$} & & & & & \\
\hline & & & Median & IQR & & & & & \\
\hline TSF & & & & & $<0.05 \dagger$ & & TSF & & $<0.05 \dagger$ \\
\hline$\leq 15$ th percentile $\|$ & 72 & 24 & 11 & $7-16$ & & -0.2 & & $-0.4-0.0$ & \\
\hline 15th-85th percentile\| & 212 & 71 & 9 & $7-13$ & & -0.2 & & $-0.5--0.1$ & \\
\hline$\geq 85$ th percentile\| & 14 & 5 & $12 \cdot 5$ & $10-14$ & & -0.9 & & $-1.6--0.2$ & \\
\hline TSF & & & & & $\mathrm{NS}^{*}$ & & & & $<0.01^{*}$ \\
\hline$<$ 50th percentile & 193 & 65 & 9 & $7-14$ & & -0.2 & & $-0.4-0.0$ & \\
\hline$\geq 50$ th percentile & 104 & 35 & 10 & $8-14$ & & -0.2 & & $-0.6--0.1$ & \\
\hline MAC & & & & & $<0.001 \dagger$ & & MAC & & NS† \\
\hline$\leq 15$ th percentile $\|$ & 52 & 18 & 13 & $9 \cdot 5-22$ & & -0.15 & & $-0.5-0.0$ & \\
\hline 15th-85th percentile\| & 235 & 79 & 9 & $7-12$ & & -0.2 & & $-0.5-0.0$ & \\
\hline$\geq 85$ th percentile $\|$ & 11 & 4 & 10 & $8-13.5$ & & -0.5 & & $-1.0--0.45$ & \\
\hline MAC & & & & & $<0.001^{\star}$ & & & & $N S^{*}$ \\
\hline$<$ 50th percentile & 175 & 59 & 11 & $8-15$ & & -0.2 & & $-0.5-0.0$ & \\
\hline$\geq 50$ th percentile & 122 & 41 & 8 & $6-11$ & & -0.2 & & $-0.5-0.0$ & \\
\hline MAMA & & & & & $<0.001 \dagger$ & & MAMA & & $<0.05 t$ \\
\hline$\leq 15$ th percentile $\|$ & 62 & 21 & 13 & $9-22$ & & -0.3 & & $-1.9-0.1$ & \\
\hline 15th-85th percentile\| & 188 & 63 & 9.5 & $7-13$ & & -0.5 & & $-1.7-0.0$ & \\
\hline$\geq 85$ th percentile\| & 48 & 16 & 8 & $6 \cdot 5-10$ & & $-1 \cdot 1$ & & $-3.2--0.1$ & \\
\hline MAMA & & & & & $<0.001^{\star}$ & & & & $N S^{*}$ \\
\hline$<50$ th percentile & 131 & 44 & 11 & $8-15$ & & -0.47 & & $-1.8-0.0$ & \\
\hline$\geq 50$ th percentile & 165 & 56 & 9 & $7-12$ & & -0.64 & & $-1.8-0.0$ & \\
\hline
\end{tabular}

Statistical analysis was performed by non-parametric * Wilcoxon $W$ test or non-parametric; $\dagger$ Median $T$ test as appropriate, for comparison of median values.

$\ddagger P$ values express statistical differences between median LOS by category of TSF, MAC, MAMA, at admission.

$\S P$ values express statistical differences between median variation of TSF, MAC and MAMA during LOS by category of TSF, MAC, MAMA, respectively.

\| Standard percentiles.

ๆ Suggested percentiles ( $<50$ th and $\geq 50$ th percentile).

Using the 50th percentile threshold, a TSF $\leq 50$ th or $>50$ th percentile did not significantly affect the median LOS. Nonetheless, a MAC or MAMA $\leq 50$ th percentile predicted longer median LOS $(P<0 \cdot 001)$; patients with TSF + MAC + MAMA $\leq 50$ th percentile also had significantly longer LOS (Table 3 ).

Comparison between the different nutritional risk and status methods in predicting a longer length of stay. In this section, we first undertook an analysis on the agreement between methods' results and LOS, by calculating the $\kappa$ concordance coefficient (Table 4). Indeed, we did find a fair and significant $\boldsymbol{\kappa}$ concordance coefficient between LOS and the methods NRS-2002, MUST, SGA, recent weight loss and MAC ( $\kappa=0.237-0.359, \quad P<0.001$ ); on the other hand, although significant, the $\kappa$ concordance coefficient between LOS and BMI/MAMA was low $(\kappa=0.069-0.163, P<0.05)$ and no agreement was found between LOS and TSF (NS).

Subsequently, to evaluate and confirm the value of the different methods tested in predicting a longer LOS, sensitivity, specificity and predictive values were calculated (Table 5).
NRS-2002, MUST, recent weight loss, SGA, MAC and MAMA presented a high sensitivity value, thus revealing a strong capacity in identify patients who had a longer LOS as at risk/undernourished; on the other hand, BMI and TSF had a low capacity to detect patients who had a longer LOS as underweight/undernourished. Specificity values were higher for SGA, MAC and MAMA, thus revealing a strong capacity to identify patients who had a shorter LOS as well nourished; NRS-2002, MUST and recent weight loss had moderate specificity values, while BMI and TSF had low specificity values, thus revealing a weak capacity in detecting patients with a shorter LOS as underweight/undernourished.

\section{Hospital stay and discharge}

Length of stay in relation to nutritional status changes during hospital stay. At discharge, the various methods showed an overall increase in the prevalence of undernutrition (Tables 3 and 6).

Table 4. Concordance between length of stay (LOS) and nutritional risk and status methods

\begin{tabular}{cccccccrr}
\hline & NRS-2002 & MUST & SGA & BMI $\left(\mathrm{kg} / \mathrm{m}^{2}\right)$ & Recent weight loss $(\mathrm{kg})$ & TSF† & MAC $\dagger$ & MAMA $\dagger$ \\
\hline LOS & $0.349^{* \star *}$ & $0.322^{* * *}$ & $0.359^{* * *}$ & $0.069^{*}$ & $0.341^{* * *}$ & $-0.065 \mathrm{NS}$ & $0.237^{* * *}$ & $0.163^{* *}$ \\
\hline
\end{tabular}

NRS-2002, Nutritional Risk Screening-2002; MUST, Malnutrition Universal Screening Tool; SGA, Subjective Global Assessment; TSF, triceps skinfold; MAC, mid-arm circumference; MAMA, mid-arm muscle area.

${ }^{\star} P<0.05,{ }^{* *} P<0.01,{ }^{* \star *} P<0.001$.

$\dagger$ In order to enable concordance calculation between TSF/MAC/MAMA and LOS, the suggested percentiles categorisation ( $<50$ th and $\geq 50$ th percentile) was used. 
Table 5. Nutritional risk and status tools and length of stay (LOS): sensitivity, specificity, positive and negative predictive values (Percentages and $95 \%$ confidence intervals)

\begin{tabular}{|c|c|c|c|c|c|c|}
\hline & \multicolumn{6}{|c|}{ LOS } \\
\hline & \multicolumn{2}{|c|}{ Sensitivity } & \multicolumn{2}{|c|}{ Specificity } & \multirow[b]{2}{*}{ PPV (\%) } & \multirow[b]{2}{*}{ NPV $(\%$} \\
\hline & $\%$ & $95 \% \mathrm{Cl}$ & $\%$ & $95 \% \mathrm{Cl}$ & & \\
\hline NRS-2002* & 0.88 & $0.85,0.92$ & 0.45 & $0.35,0.42$ & 0.75 & 0.45 \\
\hline MUST* $^{*}$ & 0.91 & $0.87,0.94$ & 0.69 & $0.63,0.72$ & 0.81 & 0.62 \\
\hline Recent weight loss $(\mathrm{kg})^{\star}$ & 0.89 & $0.86,0.92$ & 0.71 & $0.69,0.74$ & 0.87 & 0.65 \\
\hline$S_{G A}^{*}$ & 0.85 & $0.79,0.87$ & 0.93 & $0.87,0.95$ & 0.86 & 0.66 \\
\hline BMI $\left(\mathrm{kg} / \mathrm{m}^{2}\right)$ & 0.29 & $0.26,0.32$ & 0.27 & $0.23,0.30$ & 0.23 & 0.29 \\
\hline TSF $<50$ th percentile & 0.25 & $0.23,0.31$ & 0.23 & $0.21,0.27$ & 0.22 & 0.25 \\
\hline MAC $<50$ th percentile* & 0.89 & $0.87,0.96$ & 0.93 & $0.87,0.96$ & 0.81 & 0.45 \\
\hline MAMA < 50 th percentile* & 0.89 & $0.87,0.96$ & 0.93 & $0.87,0.96$ & 0.81 & 0.42 \\
\hline
\end{tabular}

PPV, positive predictive value; NPV, negative predictive value; NRS-2002, Nutritional Risk Screening-2002; MUST, Malnutrition Universal Screening Tool; SGA, Subjective Global Assessment; TSF, triceps skinfold; MAC, mid-arm circumference; MAMA, mid-arm muscle area.

* Statistically significant.

Percentage weight loss and BMI. Adding the in-hospital weight loss, there was an increase in the prevalence of patients who lost $\geq 5 \%$ of their usual weight. Moreover, twenty-nine (25\%) patients who at admission had reported $<5 \%$ weight loss, lost weight during hospital stay. In fact, a median $3 \cdot 3$ $(1.9-4.9) \%$ weight loss was accounted for in 264 (87\%) patients; of these, fifty-nine (22\%) lost $\geq 5 \%$ weight. It is worth mentioning that patients admitted for either non-elective surgery or GI surgery presented a greater in-hospital \% weight loss $(P<0.01)$. There was a positive and significant correlation between cumulative $\%$ weight loss and LOS $(0 \cdot 43, P<0 \cdot 001)$. In addition, patients who lost $\geq 5 \%$ of their weight in the hospital had a significantly longer LOS than those who lost $<5 \%$ (14 $(11-23.5) v .9(7-12) \mathrm{d}, P<0.001)$.

It is noteworthy that while in hospital, weight loss occurred in thirteen of the seventeen already underweight patients, in 169/191 (89\%) with regular BMI and in 82/90 (91\%) with overweight/obesity. Thereby, there was an increase in the undernutrition prevalence after admission: seventeen patients with regular BMI became underweight and twenty-five overweight/obese patients presented a regular BMI at discharge. Reinforcing admission data, underweight patients had a more prolonged LOS $v$. those with regular BMI or overweight/ obesity $(P<0 \cdot 001)$.
Anthropometry. Again, both standard percentiles ${ }^{(21)}$ and the 50th percentile threshold were tested in association with LOS (Table 3). A TSF $\leq 15$ th or $\leq 50$ th percentile did not affect LOS (NS); conversely, patients with a MAC or MAMA $\leq 15$ th or $\leq 50$ th percentiles had significantly longer LOS $v$. those with a MAC or MAMA $\geq 15$ th or $>50$ th percentiles.

TSF at discharge showed a median negative variation of 0.3 (interquartile range: $-0.7,-0.2) \mathrm{mm}$ in $216(72 \%)$, thus expressing a reduction in fat stores; patients with a negative TSF variation had longer LOS than those who maintained/ increased their TSF $(8(6-12) v \cdot 10(8-14) \mathrm{d}, P<0 \cdot 001)$. There was a negative correlation between TSF variation and LOS $(-0 \cdot 25, P<0 \cdot 001)$, though TSF variation during hospital stay was not associated with diagnoses or type of surgery.

Concerning MAC, 185 (62\%) patients had a median negative variation of -0.5 (interquartile range $-1.0 ;-0.2) \mathrm{mm}$; these had a longer LOS $v$. patients who maintained/increased MAC $(11(8-15) v .8(7-11) \mathrm{d}, P<0 \cdot 001)$. MAC variation and LOS were negatively correlated $(-0.35, P<0 \cdot 001)$; patients admitted for GI surgery had a greater negative MAC decrease $(P<0 \cdot 001)$.

At discharge, MAMA had decreased in 189 (63\%) patients with a median negative variation of -1.5 (interquartile range $-3 \cdot 0 ;-0 \cdot 6$ ). Similarly to TSF and MAC, the median LOS of patients with a negative MAMA variation was longer than in patients who maintained/increased MAMA (11 (8-12)

Table 6. Comparison of median hospital length of stay (LOS), according to nutritional status categories of \% weight loss and BMI at discharge

(Number of patients and percentages; medians and interquartile ranges (IQR))

\begin{tabular}{|c|c|c|c|c|c|}
\hline \multirow[b]{2}{*}{ Nutritional status categories } & \multirow[b]{2}{*}{$n$} & \multirow[b]{2}{*}{$\%$} & \multicolumn{2}{|c|}{$\operatorname{LOS}(d)$} & \multirow[b]{2}{*}{$P$} \\
\hline & & & Median & IQR & \\
\hline Cumulative weight loss (kg) & & & & & $<0.001^{*}$ \\
\hline$<5 \%$ & 90 & 30 & 9 & $7-12$ & \\
\hline$\geq 5 \%$ (undernutrition) & 208 & 70 & 14 & $11-23.5$ & \\
\hline BMI $\left(\mathrm{kg} / \mathrm{m}^{2}\right)$ & & & & & $<0.001 \dagger$ \\
\hline Underweight & 31 & 10 & 15 & $10 \cdot 5-25 \cdot 5$ & \\
\hline Normal weight & 199 & 67 & 9 & $7-13$ & \\
\hline Overweight/obese & 68 & 23 & 9 & $7-11.5$ & \\
\hline
\end{tabular}

Statistical analysis was performed by non-parametric * Wilcoxon $W$ test or non-parametric; † Median $T$ test as appropriate, for median values comparison. $P$ values express statistical differences between median LOS by category of nutritional risk and status. 
$v .8(7-11.5) \mathrm{d}, P<0.001)$ and there was a negative correlation between MAMA variation and longer LOS $(-0 \cdot 27, P<0 \cdot 001)$. By analysing the combination of TSF + MAC + MAMA, patients with a simultaneous negative variation in all three criteria $(n$ $158,53 \%)$ had a longer LOS than in patients with TSF + MAC + MAMA positive variation, $(11(8-15) v .8(7-12) \mathrm{d}, P<0 \cdot 001)$.

Nutritional risk at discharge. Given the results on nutritional deterioration during hospital stay, nutritional risk at discharge and LOS were assessed. Patients were at risk if $\%$ weight loss was $\geq 5 \%$. Patients at risk at discharge had a longer LOS then patients with no risk $(P<0 \cdot 001)$. Moreover, patients at risk presented a higher $\%$ weight loss variation, when compared to patients without risk $(3.6(2 \cdot 1-5 \cdot 4)$ v. 1.6 $(0 \cdot 8-2 \cdot 9) \%, P<0 \cdot 001)$. In what concerns anthropometric data, patients at risk had a greater TSF, MAC and MAMA negative variation $(P<0 \cdot 001)$.

In order to evaluate the relation between nutritional risk at discharge by $\geq 5 \%$ weight loss and patients' nutritional status, assessed by TSF, MAC and MAMA, a frequency analysis was performed. Results revealed a significant association between $\geq 5 \%$ weight loss $v$. MAC and MAMA percentile categories $(P<0 \cdot 001)$. Most patients with MAC or MAMA $\leq 15$ th percentile were identified as being at nutritional risk; however, by using the standard percentile categories, patients at risk and without risk were more likely to present a MAC or MAMA between the 15 th and 85th percentiles. On the other hand, using the 50th percentile as a cut-off, most patients identified at nutritional risk had a MAC or MAMA < 50th percentile, whereas patients without nutritional risk were more likely to have a MAC or MAMA $\geq 50$ th percentile $(P<0 \cdot 001)$. No association was found between nutritional risk at discharge and TSF percentile categories (NS).

\section{Discussion}

Undernutrition at admission and nutritional deterioration during hospitalisation have a detrimental effect on the clinical outcome of surgical patients, leading to an increased risk of complications and prolonged hospital $\operatorname{LOS}^{(23)}$. The present study explored the ability of various methods to assess nutritional risk and status at admission, as well as nutritional changes throughout hospital stay and their association with LOS. For anthropometry (TSF, MAC, MAMA), the 50th percentile as a unique cut-off for undernutrition was tested in addition to the standard percentiles ${ }^{(21)}$. All tested methods but TSF and BMI, as well as the 50th percentile threshold for MAC and MAMA, were found to predict a longer LOS in at-risk or already undernourished surgical patients.

The present data in surgical patients showed that poorer scores at admission of NRS-2002 or MUST as well as worse SGA and greater \% weight loss predicted longer LOS; these results agree with other studies ${ }^{(9,10,24)}$. Underweight patients by BMI also had longer LOS, however, with limited clinical value since it failed to recognise many undernourished patients; moreover, BMI had low sensitivity, specificity and predictive values in predicting LOS. BMI does not value changes in weight or in body compartments ${ }^{(25)}$; we have already demonstrated that when compared with standard methods, BMI has low sensitivity, specificity, positive and negative predictive values $^{(2,26)}$.

Body composition assessment provides more accurate information on nutritional status ${ }^{(25)}$; anthropometry is a simple, non-invasive and affordable method to assess muscle and fat depletion ${ }^{(14)}$, and thus more likely to mirror changes in body composition. The current anthropometric percentiles easily identified severe undernutrition ${ }^{(4)}$; however, they underestimate the prevalence of undernutrition ${ }^{(15,16)}$. Indeed, in our study, two-thirds of undernourished patients identified by SGA were classified as well nourished by anthropometric methods. In clinical practice, appropriate thresholds are due to identify undernutrition; we did test the 50th percentile as the cut-off and a significantly higher number of undernourished patients were identified in concordance with the other methods; in addition, a MAC/MAMA $\leq 50$ th percentile did effectively predict a longer LOS, alike the $\leq 15$ th percentile. Only muscle mass deterioration stood out as clinically significant, which concurs with a study that used bioelectrical impedance and found an association between muscle mass depletion at admission and longer LOS ${ }^{(27)}$. The 15 th or 50 th TSF percentiles were never associated with LOS, while a TSF indicative of excess fat mass ( $\geq 85$ th percentile) predicted longer LOS; excess of body fat has been recently associated with poorer outcomes ${ }^{\text {(28) }}$

The prevalence of weight loss in the hospital was extremely high, $87 \%$; weight loss during hospitalisation has been reported $^{(1,14,15,29)}$. At discharge, our data showed that a greater $\%$ weight loss predicted longer LOS and was associated with depletion of muscle and fat. Most patients were already undernourished at admission, and a continued muscle mass deterioration associated with weight loss resulted in a higher prevalence of undernutrition at discharge. Several factors have been identified as potential contributors to in-hospital weight loss, namely post-surgery catabolic stress response, underlying disease, insufficient oral intake due to anorexia, impaired cognitive function or patients' dissatisfaction with hospital meals, and inadequate perioperative nutritional care, such as prolonged and unjustified pre- and post-operative fasting and/or insufficient energy and protein supply ${ }^{(29,30)}$.

Clinicians must realise that both undernutrition at admission and nutritional deterioration in the hospital may weaken postoperative and postpone full recovery; to our knowledge, there are no studies evaluating the impact of undernutrition on after-discharge recovery of surgical patients.

Nutritional screening is clearly established as the first step in providing quality of nutritional care in hospitals ${ }^{(8,31)}$. Our results in surgical patients show that NRS-2002/MUST indicative of nutritional risk, a \% weight loss/SGA indicative of undernutrition, or the 50th percentile for MAC or MAMA effectively predicted longer LOS. Nutritional status deterioration during hospitalisation is worrying, yet preventable: the implementation of adequate perioperative nutritional care, such as the Enhanced Recovery After Surgery programme, minimises catabolism and supports anabolism, thus reducing morbidity and $\operatorname{LOS}^{(13,32)}$. Indeed, the high prevalence of patients at risk/undernutrition at admission/discharge stresses the urgent integration of screening for all surgical patients, enabling an adequate referral to nutrition professionals for a comprehensive 
assessment and individualised intervention integrated in the overall management; this is the only effective approach, allowing for an adequate nutritional intake before and after discharge, and potentially promoting a faster recovery.

\section{Acknowledgements}

The present study was partially supported by a Grant from the 'Fundação para a Ciência e Tecnologia' (RUN 437). All authors hereby declare that there were no financial and personal relationships with other people or organisations that could inappropriately influence their work. A. I. A. was responsible for the data analysis, interpretation and writing of the manuscript. M. Correia was responsible for data collection. M. Camilo was responsible for the writing and final review of the manuscript as well as data interpretation. P. R. was responsible for the writing and final review of the manuscript, data interpretation and data analysis.

\section{References}

1. Brunn LI, Bosaeus I, Bergstad I, et al. (1999) Prevalence of malnutrition in surgical patients: evaluation of nutritional support and documentation. Clin Nutr 18, 141-147.

2. Almeida AI, Correia M, Camilo M, et al. (2012) Nutritional risk screening in surgery: valid, feasible, easy! Clin Nutr 31, 206-211.

3. Correia M, Caiaffa W, Lázaro da Silva A, et al. (2001) Risk factors for malnutrition in patients undergoing gastroenterological and hernia surgery: an analysis of 374 patients. Nutr Hosp 16, 59-64.

4. Mourão F, Amado D, Ravasco P, et al. (2004) Nutritional risk and status assessment in surgical patients: a challenge amidst plenty. Nutr Hosp 9, 83-88.

5. Norman K, Pichard C, Lochs H, et al. (2008) Prognostic impact of disease-related malnutrition. Clin Nutr 27, 5-15.

6. Larsson J, Ảkerlind I, Permerth J, et al. (1994) The relation between nutritional state and quality of life in surgical patients. Eur J Surg 160, 329-334.

7. Leonard-Jones J (2002) A Positive Approach to Nutrition as Treatment. London: King's Fund Report.

8. Council of Europe Committee of Ministers (2003) Resolution ResAP (2003)3 on Food and Nutritional Care in Hospitals. Strasbourg: Council of Europe.

9. Kyle U, Kossovsky MP, Karsegard VL, et al. (2006) Comparison of tools for nutritional assessment and screening at hospital admission: a population study. Clin Nutr 25 , 409-417.

10. Raslan M, Gonzalez MC, Dias MC, et al. (2010) Comparison of nutritional risk screening tools for predicting clinical outcomes in hospitalized patients. Nutrition 26, 721-726.

11. Stratton RJ, King CL, Stroud MA, et al. (2006) 'Malnutrition Universal Screening Tool' predicts mortality and length of stay in acutely ill elderly. Br J Nutr 95, 325-330.

12. Schiesser M, Müller S, Kirchhoff P, et al. (2008) Assessment of a novel screening score for nutritional risk in predicting complications in gastro-intestinal surgery. Clin Nutr 27, 565-570.

13. Weimann A, Braga M, Harsanyi L, et al. (2006) ESPEN guidelines on enteral nutrition: surgery including organ transplantation. Clin Nutr 25, 224-244.
14. McWhirter JP \& Pennington CR (1994) Incidence and recognition of malnutrition in hospital. BMJ 308, 945-948.

15. Corish CA, Flood P, Mulligan S, et al. (2000) Apparent low frequency of undernutrition in Dublin hospital in-patients: should we review the anthropometric thresholds for clinical practice? Br J Nutr $\mathbf{8 4}, 325-335$.

16. Fettes SB, Davidson HI, Richardson RA, et al. (2002) Nutritional status of elective gastrointestinal surgery patients pre- and post-operatively. Clin Nutr 21, 249-254.

17. Kondrup J, Allison SP, Elia M, et al. (2003) ESPEN guidelines for nutritional screening 2002. Clin Nutr 22, 415-421.

18. Elia M. (2003) Screening for malnutrition: a multidisciplinary responsibility. Development and use of the Malnutrition Universal Screening Tool (MUST) for adults. Malnutrition Advisory Group, a Standing Committee of BAPEN.

19. Detsky A, McLaughlin JR, Baker JP, et al. (1987) What is subjective global assessment of nutritional status? JPEN 11, $8-13$.

20. WHO (1998) Consultation on Obesity. Geneva: World Health Organization.

21. Bloch AS \& Shills ME (editors) (2006) Part VII: appendix. Anthropometric data. In Modern Nutrition in Health and Disease, 10th ed., pp. 1939-1948 [ME Shills, M Shike, AC Ross, B Caballero and RJ Cousins, editors]. Baltimore, MD: Lippincott Williams \& Wilkins.

22. Fleiss J (1981) Statistical Methods for Rates and Proportions, 2nd ed. New York: John Wiley \& Sons.

23. Shils ME, Shike M, Ross AC, et al. (2006) Modern Nutrition in Health and Disease, 10th ed. Baltimore, MD: Lippincott Williams \& Wilkins.

24. Sorensen J, Kondrup J, Prokopowicz J, et al. (2008) EuroOOPS: an international, multicentre study to implement nutritional risk screening and evaluate clinical outcome. Clin Nutr 27, 340-349.

25. Kyle UG, Morabia A, Slosman DO, et al. (2001) Contribution of body composition to nutritional assessment at hospital admission in 995 patients: a controlled population study. Br J Nutr 86, 725-731.

26. Boléo-Tomé C, Monteiro Grillo I, Camilo M, et al. (2012) Validation of the Malnutrition Universal Screening Tool (Must) in cancer. BrJ Nutr 108, 343-348.

27. Pichard C, Kyle UG, Morabia A, et al. (2004) Nutritional assessment: lean body mass depletion at hospital admission is associated with an increased length of stay. Am J Clin Nutr 79, 613-618.

28. Kyle UG, Pirlich M, Lochs H, et al. (2005) Increased length of hospital stay in underweight and overweight patients at hospital admission: a controlled population study. Clin Nutr 24, 133-142.

29. Caccialanza R, Klersy C, Cereda E, et al. (2010) Nutritional parameters associated with prolonged hospital stay among ambulatory adult patients. CMAJ 182, 1843-1849.

30. Hiesmayr M, Schindler K, Pernicka E, et al. (2009) Decreased food intake is a risk factor for mortality in hospitalised patients: the nutritionday survey 2006. Clin Nutr 28, 484-491.

31. European Parliament Resolution of 9 October 2008 on 'Together for Health: A Strategic Approach for the EU 2008-2013'. (2010) Off J Eur Union 52, 56-64.

32. Teeuwen PH, Bleichrodt RP, Strik C, et al. (2010) Enhanced recovery after surgery (ERAS) versus conventional postoperative care in colorectal surgery. J Gastrointest Surg 14, $88-95$. 